\title{
COHOMOLOGY, DIMENSION AND LARGE RIEMANNIAN MANIFOLDS
}

\author{
A.N. DRANISHNIKOV \\ Department of Mathematics \\ University of Florida \\ P.O. Box 118105, 358 Little Hall \\ Gainesville, FL 32611-8105, USA
}

(Received April 2, 1997 and in revised form April 24, 1997)

\begin{abstract}
This paper surveys recent results on dimension and cohomology of the Higson corona of uniformly contractable manifolds
\end{abstract}

KEY WORDS AND PHRASES: Dimension, cohomology, aspherical manifolds, Higson corona, Novikov conjecture

1991 AMS SUBJECT CLASSIFICATION CODES: 54D35, 54F45, 57M10, 57S30

\section{INTRODUCTION}

There are many well-known conjectures and problems in the area of activity surrounding the Novikov Higher Signature Conjecture. Different branches of mathematics meet there such as differential geometry, algebraic topology, operator algebras, K-theory, geometric topology, geometric group theory The goal of this note is to demonstrate that dimension theory also comes into the picture

First we formulate two conjectures in the area the formulations of which is less technical. We recall that a manifold $M^{n}$ is called aspherical if its homotopy groups $\pi_{2}(M)$ are trivial in dimensions $\geq 2$ The following is a special case of the Strong Novikov conjecture [1]

GROMOV-LAWSON CONJECTURE. A closed aspherical manifold does not admit a Riemannian metric of a positive scalar curvature.

One of the strongest in the area is the following

BOREL CONJECTURE. Any two homotopy equivalent closed aspherical manifold are homeomorphic.

The standard approach in any problem about an aspherical manifold $N^{n}$ consists of taking into consideration the universal cover $M^{n}$ of $N^{n}$ together with the action of the fundamental group $\Gamma=\pi_{1}\left(N^{n}\right)$. As it follows from Whitehead theorem, the universal cover of an aspherical manifold is contractible. Since every contractible manifold $M^{n}$ crossed with the real line $\mathbf{R}$ is homeomorphic to euclidean space $\mathbf{R}^{n+1}$, without a big loss we may assume that $M^{n}$ is homeomorphic to $\mathbf{R}^{n}$. So, the problem about an aspherical manifold is a problem about a $\Gamma$-periodic metric on $\mathbf{R}^{n}$. The main feature of a $\Gamma$-periodic metric in this case is that the metric space $M=\mathbf{R}^{n}$ is uniformly contractible. We recall that a metric space $X, \rho$ is uniformly contractible if for every positive number $R$ there is a bigger number $S$ such that every ball $B_{\rho}(x, R)$ can be contracted to a point in $B_{\rho}(x, S)$.

The study of a non-compact space can be simplified by adding a corona to $M$ making $M$ compact Such a corona for metric spaces was introduced by Higson [2].

DEFINITION. If $M$ is a space and $\phi: M \rightarrow \mathbf{C}$ is a continuous function, define $V_{r}(\phi): M \rightarrow \mathbf{R}^{+}$ by 


$$
V_{r}(\phi)=\sup \left\{|\phi(y)-\phi(x)|: y \in B_{r}(x)\right\} .
$$

Then $C_{h}(M)$ is the space of all bounded continuous functions $\phi: M \rightarrow \mathbf{C}$ so that for each $r>0$, $V_{r}(\phi) \rightarrow 0$ at infinity. Lemma 5.3 of [3] proves that $C_{h}(M)$ is a $C^{*}$-algebra, so it makes sense to define the Higson compactification, $\bar{M}$ of $M$ to be the maximal ideal space of $C_{h}(M)$. Then the remainder of the Higson compactification $\nu M=\bar{M} \backslash M$ is called the Higson corona

The Higson corona was introduced for an analysis of the index theorem for noncompact manifolds $J$ Roe demonstrated in [3] that the Higson corona can be used for characterization of the notion of hypereuclidean space which is due to Gromov [4]:

A manifold $M^{n}$ is hypereuclidean if there is a map $f: \nu M^{n} \rightarrow S^{n-1}$ of the degree one of the Higson corona onto $n$ - 1-sphere

The degree of $f$ in this case is the degree of the homomorphism $\partial f^{*}: H^{n-1}\left(S^{n-1}\right)=$ $\mathbf{Z} \rightarrow \mathbf{Z}=H_{c}^{n}\left(M^{n}\right)$.

Let $\tilde{M}^{n}$ be a compactification of $M^{n}$. An action of $\Gamma$ on $M^{n}$ by isometries is called small at infinity with respect to a compactification $\tilde{M}^{n}$ if for every point $x \in \tilde{M}^{n} \backslash M^{n}$ and for every compact set $C \subset M^{n}$ for every neighborhood $U$ of $x$ there is a smaller neighborhood $V$ such if $g(C) \cup V \neq \emptyset$ for some $g \in \Gamma$, then $g(C) \subset U$. Carlsson and Pedersen proved in [5] (see also [6]) that if the universal cover $M^{n}$ admits an acyclic compactification with a small action of $\Gamma$ at infinity, then the Novikov conjecture holds for the group $\Gamma$.

PROPOSITION. Let $\tilde{M}^{n}$ be a compactification of $M^{n}$ An action of $\Gamma$ on $M^{n}$ is small at infinity with respect to a compactification $\tilde{M}^{n}$ if and only if there is a map $g: \bar{M}^{n} \rightarrow \tilde{M}^{n}$ of the Higson compactification, which is the identity of $M^{n}$.

PROOF. The 'only if case was proven in [6, Proposition 3.1].

Let $g: \bar{M}^{n} \rightarrow \tilde{M}^{n}$ be that map. Take a point $x \in \tilde{M}^{n} \backslash M^{n}$, take a compact set $C \subset M^{n}$ and a neighborhood $U$ containing $x$. There is a compact metric space $\check{M}^{n}, \rho$ and a map $q: \tilde{M}^{n} \rightarrow \check{M}^{n}$ such that the restriction $\left.q\right|_{M^{n}}=i d_{M^{n}}$ and there is an open set $U^{\prime}, q(x) \in U^{\prime}$ in $\check{M}^{n}$ with $q^{-1}\left(U^{\prime}\right) \subset U$ Let $d=\operatorname{dist}_{\rho}\left(q(x), \check{M}^{n} \backslash U^{\prime}\right)$. By Proposition 1 of [7] $\lim _{y \rightarrow \infty} \operatorname{diam}(B(y, R))=0$ for any $R$, here $B(y, R)$ is the ball in the metric space $M^{n}$ of radius $R$ and centered at $y$ Since $\Gamma$ acts on $M^{n}$ by isometries, the diameter of $g(C)$ is equal to the diameter of $C$ for all $g \in \Gamma$ Hence there is a neighborhood $O$ of the boundary $\check{M}^{n} \backslash M^{n}$ such that diam $g(C)<\frac{d}{2}$ whenever $g(C) \cup O \neq \emptyset$ Define $V=q^{-1}\left(\operatorname{Int} B_{\rho}\left(q(x), \frac{d}{2}\right) \cap O\right)$.

In [8] it is proven that the Gromov-Lawson conjecture holds for manifolds $N^{n}$ with the hypereuclidean universal cover $M^{n}$. This result makes the following conjecture natural.

GROMOV'S CONJECTURE [4] Every uniformly contractible manifold is hypereuclidean

Unfortunately this version of Gromov's conjecture is not correct.

THEOREM 1 [9]. There is a uniformly contractible metric on $\mathbf{R}^{8}$ which is not hypereuclidean

This counterexample is based on the strange phenomenon in dimension theory: cohomological and covering dimension may disagree for infinite-dimensional spaces [10]

Note that the rational version of Gromov's conjecture still may be correct. To avoid our counterexample in the integral case one should restrict oneself to uniformly contractible manifolds with bounded geometry [11]. Any of these two modified versions would be sufficient to derive the GromovLawson conjecture. According to [12, Theorem 3.1] the rational version of Gromov's conjecture is equivalent to the following

WEINBERGER CONJECTURE [3]. For every uniformly contractible manifold $X$ the boundary homomorphism $\partial H^{n-1}(\nu X ; \mathbf{Q}) \rightarrow H_{c}^{n}\left(\mathbf{R}^{n} ; \mathbf{Q}\right)$ is an epimorphism

The Weinberger conjecture can be easily verified for the standard euclidean spaces $\mathbf{R}^{n}$ and for the hyperbolic spaces $\mathbf{H}^{n}$ [6]. The stronger conjecture (see [3]) was that the Higson compactification of a 
uniformly contractible manifold is acyclic This conjecture was disproved by $\mathrm{J}$ Keesling [13] who showed that the 1-dimensional cohomology group of the Higson compactification of the euclidean space is nontrivial As it can easily be seen, to derive the Weinberger conjecture it is sufficient to have trivial only $n$-dimensional cohomology group. It turns out to be that this also is not true even for the euclidean space

THEOREM 2 [12]. $H^{n}\left(\overline{\mathbf{R}^{n}} ; \mathbf{Q}\right) \neq 0$

Nevertheless in the hyperbolic case there is an acyclicity theorem [12].

In the example of Theorem 1 the dimension of the Higson corona $\nu M^{8}$ is infinite [6] Perhaps this is the main obstruction for a uniformly contractible manifold to be hyperspherical.

PROBLEM. Does the Gromov-Lawson Conjecture (the Novikov Conjecture) hold for manifolds $N^{n}$ with finite dimension of the Higson corona of the universal cover. $\operatorname{dim} \nu M^{n}<\infty$ ?

The importance of this question is supported by the following two results.

THEOREM 3 [14] If asdim $M^{n}<\infty$ then the Gromov-Lawson Conjecture (and the Novikov Conjecture) holds for $M^{n}$

We recall that asdim stands for the asymptotic dimension introduced by Gromov [15]. By the definition $\operatorname{asdim} M^{n} \leq n$ if for every positive $R>0$ there exists a uniformly bounded covering $\mathcal{U}$ such that every $R$-ball $B(x, R)$ in $M^{n}$ intersects no more than $n+1$ elements of $\mathcal{U}$.

THEOREM 4 [6]. For every proper metric space $M$ there is the inequality $\operatorname{dim} \nu M \leq \operatorname{asdim} M$

In the conclusion we formulate the classical Novikov conjecture.

NOVIKOV CONJECTURE. Let $G_{n}^{k}$ be the Grassmanian space of $k$-dimensional oriented vector subspaces in $n$-space with the natural topology. There is the natural imbedding $G_{n}^{k} \subset G_{n+1}^{k}$. Then one can define the space $G_{\infty}^{k}=\lim \neg G_{n}^{k}$. The natural imbedding $G_{\infty}^{k} \subset G_{\infty}^{k+1}$ leads to the definition of the space $B O=G_{\infty}^{\infty}=\lim _{\hookrightarrow} G_{\infty}^{k}$. The tangent bundle of an $n$-dimensional manifold $N$ can be obtained as the pull-back from the natural $n$-bundle over the space $G_{\infty}^{n}$. Let $f_{\tau}: N \rightarrow B O$ be a map which induces the tangent bundle on $N$. The cohomology ring $H^{*}(B O ; \mathbf{Q})$ is a polynomial ring generated by some elements $a_{\imath} \in H^{4 \imath}(B O ; \mathbf{Q})$. The rational Pontryagin classes of a manifold $N$ are the elements $p_{\imath}=f^{*}\left(a_{\imath}\right) \in H^{4 \imath}(B O ; \mathbf{Q})$. Novikov [16] proved that the rational Pontryagin classes are topological invariants. It was known that they are not homotopy invariants. Hirzebruch found polynomial $L_{k}\left(p_{1}, \ldots, p_{k}\right) \in H^{4 i}(N ; \mathbf{Q})$ which do not depend on $N$ and such that the signature of every closed (oriented) $4 k$ manifold $N$ can be defined as the value of $L_{k}$ on the fundamental class of $N$. Note that the signature is homotopy invariant and even more, it is bordism invariant. For non-simply connected manifolds Novikov defined the higher signature as follows Let $\Gamma$ be the fundamental group of a closed oriented manifold $N$, let $g: N \rightarrow B \Gamma=K(\Gamma, 1)$ be a map classifying the universal cover of $N$ and let $b \in H^{*}(K(\Gamma, 1) ; \mathbf{Q})$. Then he defines the b-signature as $\operatorname{sign}_{b}(N)=\left\langle L_{k} \cap g^{*}(b),[N]\right\rangle$, here $4 k+\operatorname{dim}(b)=\operatorname{dim} N$.

CONJECTURE. Let $h: N \rightarrow M$ be an orientation preserving homotopy equivalence between two close oriented manifolds, then $\operatorname{sign}_{b}(N)=\operatorname{sign}_{b}(M)$ for any $b \in H^{*}(K(\Gamma, 1) ; \mathbf{Q})$.

We say that the Novikov conjecture holds for a group $\Gamma$ if it holds for every manifold with the fundamental group $\Gamma$. It is known that the Novikov conjecture holds for $\Gamma$ if and only if the certain homomorphism from the surgery exact sequence [17],[18],[19], called the assembly map [20]

$$
A: H_{*}(K(\Gamma, 1) \mathbf{Q}) \rightarrow L_{*}(\mathbf{Z}[\Gamma]) \otimes \mathbf{Q}
$$

is an injection. The Novikov conjecture is verified for abelian groups, hyperbolic groups, CAT(0)groups, discrete subgroups of connected Lie groups and others [4],[6],[16],[21],[22],[23],[24],[25],[26]

ACKNOWLEDGEMENT. The author was partially supported by NSF grant DMS-9626101. 


\section{REFERENCES}

[1] ROSENBERG, J., $C^{*}$-algebras, positive scalar curvature and the Novikov conjecture, Publ. I.H.E.S. 58 (1983), 409-424

[2] HIGSON, N., On the Relative K-homology Theory of Baum and Douglas, Preprint (1990)

[3] ROE, J., Coarse cohomology and index theory for complete Riemannian manifolds, Memoirs, Amer. Math. Soc. 497 (1993)

[4] GROMOV, M., Large Riemannian manifolds, Lecture Notes in Math. 1201 (1985), 108-122

[5] CARLSSON, G. and PEDERSEN, E., Controlled algebra and the Novikov conjecture for $K$ and $L$ theory, Topology 34 (1995), 731-758.

[6] DRANISHNIKOV, A.N., KEESLING, J.E. and USPENSKIJ, V.V, On the Higson Corona of Uniformly Contractible Spaces, Preprint (1996).

[7] KASPAROV, G.G., Equivariant $K K$-theory and the Novikov conjecture, Invent. Math. 91 (1988), 147-201

[8] GROMOV, M. and LAWSON, H.B., Positive scalar curvature and the Dirac operator, Publ. I.H.E.S. 58 (1983), 83-196.

[9] DRANISHNIKOV, A.N., FERRY, S. and WEINBERGER, S., Large Riemannian Manifolds Which are Flexible, Preprint (1994)

[10] DRANISHNIKOV, A.N., Homological dimension theory, Russian Mathematical Surveys 43 (1988), 11-63.

[11] HIGSON, N. and ROE, J., The Baum-Connes conjecture in coarse geometry, LMS Lecture Notes 227 (1995), 227-254.

[12] DRANISHNIKOV, A.N and FERRY, S., The Higson-Roe Corona, Preprint (1997).

[13] KEESLING, J., The one-dimensional Cech cohomology of the Higson compactification and its corona, Topology Proceedings 19 (1994), 129-148

[14] YU, G., The Novikov Conjecture and Groups With Finite Asymptotic Dimensions, Preprint (1995)

[15] GROMOV, M., Asymptotic invariants for infinite groups, LMS Lecture Notes 182(2) (1993)

[16] NOVIKOV, S.P., On manifold with free abelian fundamental group and applications (Pontryagin classes, smoothings, high-dimensional knots), Izv. Akad. Nauk SSSR 30 (1966), 208-246.

[17] KAMINIKER, J. and MILLER, J.G., A comment on the Novikov conjecture, Proc. Amer. Math. Soc. 83:3 (1981), 656-658.

[18] WALL, C.T.C., Surgery on Compact Manifolds, Academic Press, New York, 1970.

[19] RANICKI, A.A., Algebraic $L$-theory and topological manifolds, Cambridge Tracts in Mathematics 102, Cambridge University Press, 192.

[20] ROSENBERG, J., Analytic Novikov for topologists, LMS Lecture Notes 226 (1995), 338-372

[21] MISCHENKO, A.S., Homotopy invariants of nonsimply connected manifolds. Rational invariants, Izv. Akad. Nauk SSSR 30:3 (1970), 501-514.

[22] CAPPELL, S.E., On homotopy invariance of high signatures, Invent. Math. (1976), 171-179

[23] FARRELL, F.T. and HSIANG, W.-C., On Novikov conjecture for nonpositively curved manifolds, Ann. Math. 113 (1981), 197-209.

[24] CONNES, A. and MOSCOVICI, H., Cyclic cohomology, the Novikov conjecture and hyperbolic groups, Topology 29:3 (1990), 345-388.

[25] FERRY, S. and WEINBERGER, S., A coarse approach to the Novikov Conjecture, LMS Lecture Notes 226 (1995), 147-163.

[26] KASPAROV, G.G. and SKANDALIS, G., Groups acting on buildings, operator $K$-theory, and Novikov conjecture, K-theory 4 (1991), 303-337. 


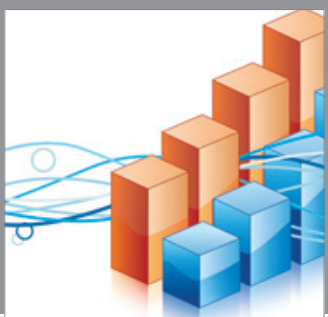

Advances in

Operations Research

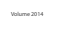

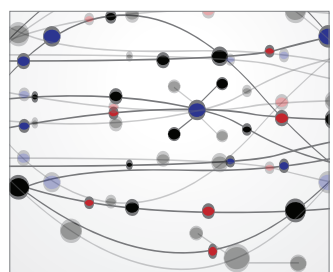

\section{The Scientific} World Journal
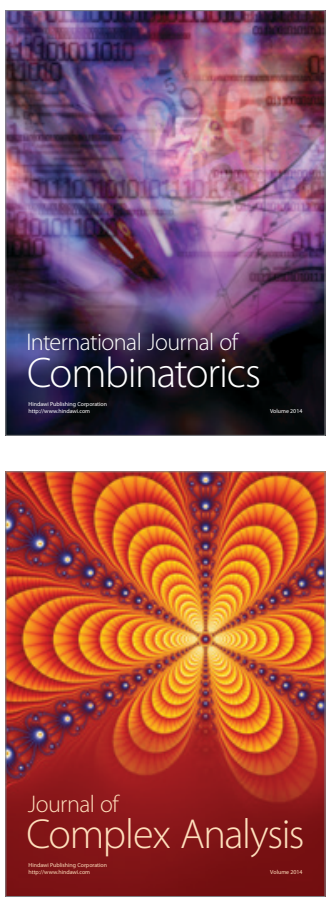

International Journal of

Mathematics and

Mathematical

Sciences
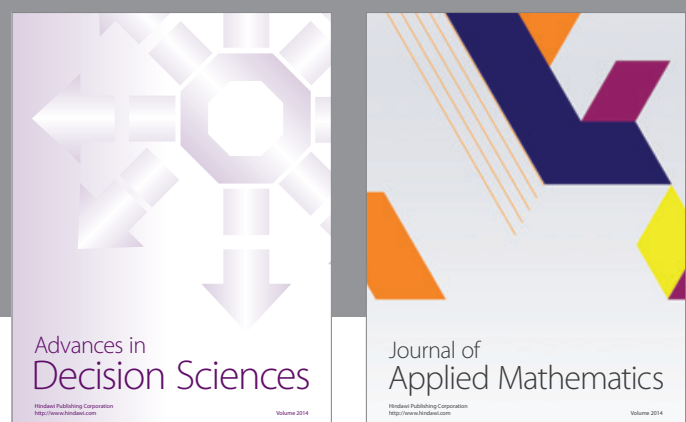

Journal of

Applied Mathematics
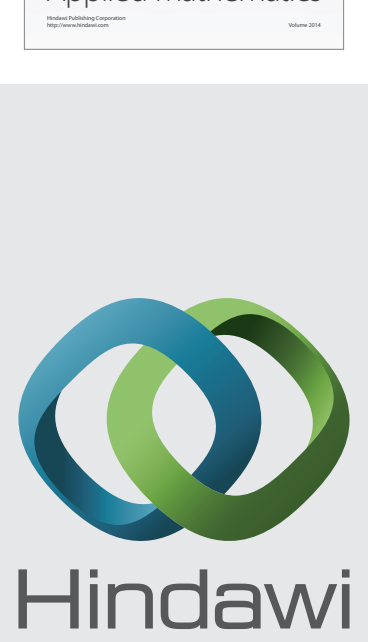

Submit your manuscripts at http://www.hindawi.com
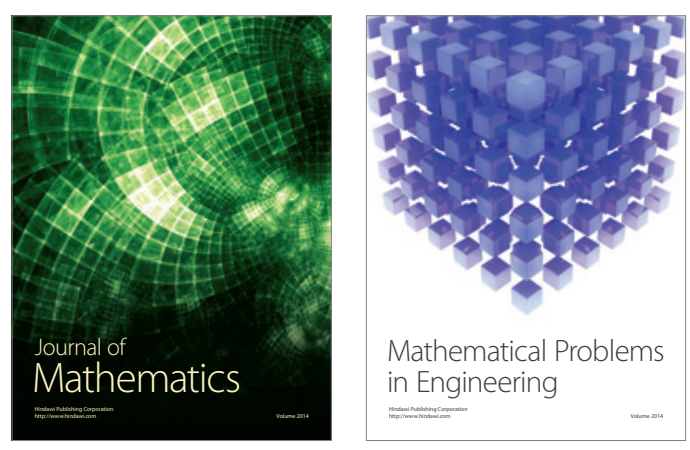

Mathematical Problems in Engineering
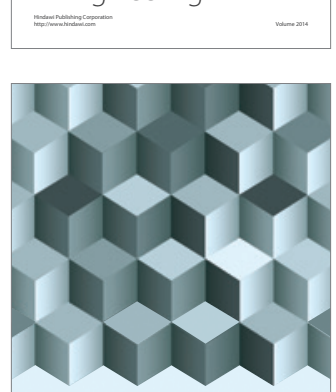

Journal of

Function Spaces
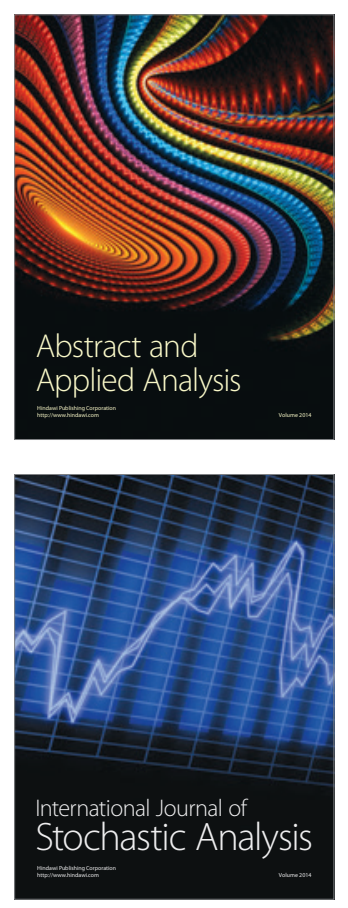

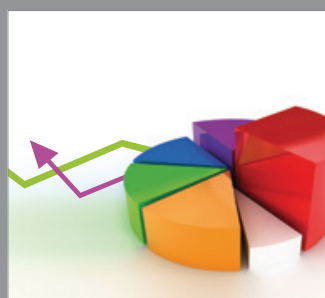

ournal of

Probability and Statistics

Promensencen
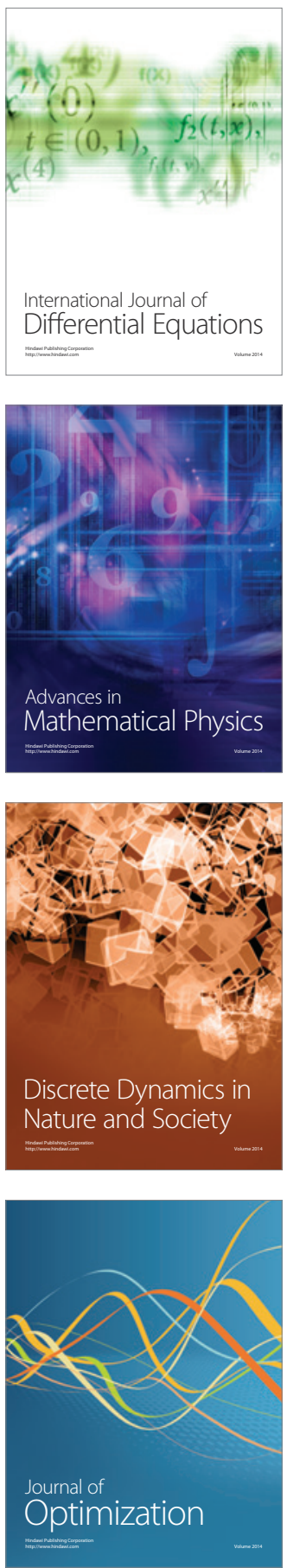